\title{
RECHERCHES SUR LA SPÉCIFICITÉ PARASITAIRE DES COCCIDIES
}

\author{
Par Charles CORCUFF
}

\section{Spécificité parasitaire des Coccidies}

La spécificité parasitaire des coccidies est fort intéressante à préciser car elle est à la base de toute prophylaxie des coccidioses tant humaines qu'animales. En effet, il est important de savoir si la coccidie hébergée par tel ou tel animal est capable d'infecter l'homme ; dans ce cas, l'animal en question devient un réservoir de virus et doit être traité comme tel. Nous voyons là un des aspects du problème général de la spécificité parasitaire. Or ce problème, agité par de nombreux auteurs, envisagé de maintes façons, est resté dans bien des points assez obscur. Nous allons voir quelle opinion on peut être amené à se faire en ce qui concerne le groupe des coccidies.

La première coccidie fut trouvée chez le lapin. Leuwenhoeck, à en croire Dobell, avait dès le xvir siècle noté la présence d'oocystes dans la bile de cet animal ; mais c'est en 1839 seulement que Hake en donna une description ; il les prit toutefois pour des globules de pus ; les auteurs suivants ne tardèrent pas cependant à reconnaître la nature parasitaire des oocystes trouvés tant dans le foie que dans l'intestin. Bien que Leuckart eût considéré comme distinct le parasite du foie et celui de l'intestin, d'autres auteurs voulurent y voir au contraire une seule espèce ; en outre on rapporta à cette espèce avec la plus grande facilité, et sans autre recherche, des oocystes de coccidie rencontrés chez l'homme ou chez d'autres mammifères. Dans l'opinion de beaucoup d'auteurs, "la coccidie du lapin " fut pendant longtemps la coccidie par excellence, la seule du genre Eimeria et capable d'être rencontrée un peu partout:

On connaissait d'autre part chez le chien et le chat une coccidie appartenant celle-là au genre Isospora. Eimer signala chez l'homme une coccidie analogue. Il fut admis longtemps que le parasite humain n'était autre que le parasite animal.

Les cas de coccidiose humaine, signalés par la suite, d'ailleurs

Annales de Parasitologie, T. VI, $\mathrm{N}^{\circ} 4 .-1^{\mathrm{er}}$ octobre 1928, p. 404-418. 
fort rares et généralement peu précis, furent ainsi considérés de façon générale comme des infections accidentelles de l'homme par. des parasites d'animaux.

Mais survint avec la guerre l'habitude des examens microscopiques des selles ; ceux-ci montrèrent que l'homme présentait beaucoup plus fréquemment qu'on ne pensait des oocystes de coccidie. Dobell (1919) reprenant alors l'étude des cas précédemment connus fut amené à la conclusion que toutes les coccidies signalées chez l'homme étaient vraisemblablement particulières à l'homme et qu'il n'y avait aucune preuve de leur origine animale. Wenyon, en 1922, étudia de près les coccidies des chiens et des chats et leur rapport avec la coccidie humaine; il montra que les Isospora des chiens et des chats étaient communes à ces deux espèces, qu'elles étaient plus nombreuses qu'on ne pensait, et enfin que l'Isospora humaine, que l'on rencontrait assez fréquemment, en était nettement différente.

En ce qui concerne également les coccidies des animaux, la même incertitude à l'égard de leur spécificité que pour les coccidies de l'homme a régné pendant longtemps : c'est ainsi que tandis que certains auteurs considéraient la coccidie des bovidés comme une espèce spéciale Eimeria zurnii, d'autres ne voulaient voir en elle que " la coccidie " du lapin; de même on rapporta la coccidie du mouton à celle du bœuf.

C'était en somme nier toute spécificité parasitaire et aboutir à la confusion la plus complète. Il faut cependant reconnaître que de tout temps certains auteurs, généralement non suivis, soutinrent l'opinion pluraliste ; et c'est celle-ci qui, grâce aux travaux récents, tend maintenant à prévaloir, encore que la question soit loin d'être élucidée dans tous ses détails.

Cette confusion d'ailleurs se comprend facilement si l'on examine d'un peu plus près les bases que l'on peut avoir pour se faire une opinion. Dans la plupart des cas, le diagnostic de la coccidie a porté sur l'oocyste tel qu'on le rencontre dans les selles. Or dans cet état tous les oocystes se ressemblent beaucoup et on a entre eux fort peu de points de comparaison ; certains, il est vrai, sont de forme sphérique, d'autres sont ovalaires ; mais ce n'est pas absolu et dans la même espèce on peut rencontrer des oocystes ayant les deux formes, tandis que deux espèces différentes peuvent présenter des oocystes de forme semblable.

On peut noter également des différences de taille et c'est là un argument qui a été souvent invoqué ; mais il ne faut pas oublier que cet argument n'est valable que si cette différence est nettement tranchée et constante; certaines espèces comme l'Eimeria du 
chien par exemple (1) sont susceptibles de fournir des oocystes dont la taille varie du simple au double; des espèces considérées actuellement comme différentes ont par contre des oocystes de même taille. L'étude des dimensions, des caractères dits biométriques entraîne d'ailleurs un travail fort long, extrêmement fastidieux, toujours incomplet cependant et illusoire dans ses résultats, au moins dans la grande majorité des cas. Quant aux différences portant sur la coloration, la présence ou l'absence de micropyle, ce sont là des caractères plutôt individuels, d'observation inconstante et souvent difficile sur lesquels on ne saurait tabler. On voit combien il est risqué de tirer des conclusions nettes de l'examen des oocystes tels qu'ils sont émis. Or beaucoup de chercheurs se sont contenté de ce seul examen et ont basé leur opinion sur cette méthode simpliste.

La connaissance du cycle évolutif de. la coccidie est susceptible de fournir des précisions nouvelles. Le cycle schizogonique intracellulaire, difficile à suivre et loin d'ailleurs d'être entièrement connu pour toutes les coccidies, n'a pour ainsi dire jamais été utilisé comme élément de différenciation entre les espèces ; tout au plus pourrait-on dans cet ordre d'idées, citer les travaux de Waworuntu sur les coccidies du lapin. Le cycle sporogonique, par contre, est facile à observer, car on obtient aisément la segmentation complète des oocystes. Or, si l'on suit avec soin cette segmentation, on peut noter dans la façon dont elle se fait certaines différences non seulement d'après les genres, mais encore, dans le même genre, suivant les espèces ; ces différences portent surtout sur les reliquats de segmentation, masses granuleuses qui peuvent persister dans l'oocyste après formation des spores, ou dans le sporocyste après formation des sporozoïtes. Ces masses peuvent être absentes, être très réduites ou au contraire très abondantes ; mais dans une même espèce elles présentent toujours un même aspect. Il y a donc là un caractère facile à observer, dont on a longtemps méconnu la valeur et dont nous verrons plus loin l'application à la coccidie du lapin.

Enfin il reste encore un procédé de différenciation des espèces, c'est le moyen biologique de l'immunité croisée : tenter d'infecter tel animal avec une coccidie provenant de tel autre, et réciproquement. C'est dans cette voie surtout que se sont faites les recherches. Mais si l'on veut que celles-ci soient fructueuses, il faut s'entourer d'un grand nombre de précautions. On n'aura certes aucune difficulté à obtenir la maturation des oocystes, mais il faut tout d'abord

(1) Peut être $y$ a-t-il là plusieurs espèces; c'est un point qui n'est pas encore tranché. 
être bien sûr do l'espèce coccidienne avec laquelle on opère ; il faut attendre suffisamment pour juger de l'infection éventuelle de l'animal expérimenté ; il faut surtout être sûr, en cas d'infection, de n'avoir pas affaire à une coccidiose déjà établie chez l'animal avant l'expérience ; les coccidioses spontanées sont en effet fréquentes chez presque tous les mammifères ; elles sont parfois fort discrètes et difficiles à déceler ; il est pourtant absolument nécessaire de les éliminer si l'on veut que l'expérience faite ait une portée suffisante pour conclure.

D'autre part il semble que généralement une coccidiose antérieure confère un certain degré d'immunité et si l'expérience n'a pas produit d'infection, la possibilité d'une atteinte antérieure laissera toujours subsister un doute sur la valeur du résultat. Aussi est-on amené à opérer sur des animaux jeunes, qui sont d'ailleurs plus susceptibles à une infection et ces animaux doivent avoir été élevés d'une façon spéciale afin d'être à l'abri de toute contamination spontanée ; en réalité il est extrêmement difficile d'obtenir des élevages absolument indemnes; c'est ce qui rend si malaisées ces expériences d'immunité croisée qui du point de vue théorique paraissent si faciles. La valeur de ces expériences dépend donc avant tout de la rigueur des conditions dans lesquelles elles ont été faites; il y a là un point qu'il ne faut pas perdre de vue lorsque l'on a à discuter les conclusions qui en sont tirées.

Il ne sera souvent pas de trop de toutes les méthodes pour arriver à bien établir la valeur spécifique d'une espèce coccidienne. On ne saurait donner un plus bel exemple des résultats acquis ainsi que celui des coccidies du lapin.

Dès 1879, Leuckart distinguait pour celles-ci deux espèces, l'une parasite du foie, l'autre parasite de l'intestin. Mais peu d'années après, la majorité des auteurs rejetait cette opinion pour admettre l'unicité. Plus tard, Railliet et Lucet reprenaient la théorie de Leuckart et apportaient des caractères nouveaux pour différencier les deux parasites, caractères portant sur la segmentation de l'oocyste et sur les lésions obtenues par l'infection de lapins indemnes. Néanmoins Railliet et Lucet ne furent pas suivis et l'on persista à ne voir qu'une espèce: Eimeria stiedai. Reichenow en 1921, reprenant cette étude, fut amené à admettre la dualité mais il semble que dans ses recherches il y ait eu certaines causes d'erreur dues à des infections mixtes méconnues et ses conclusions ne pouvaient par suite être jugées définitives. Les travaux récents de Pérard ont maintenant définitivement tranché la question. Avec les plus grandes difficultés cet auteur réussit à obtenir des infections pures; il put ainsi montrer qu'il y avait entre les deux espèces des 
différences de taille de l'oocyste, des différences dans le mode de segmentation de celui-ci (la coccidie intestinale donne toujours un reliquat de segmentation alors que l'autre n'en donne pas) et enfin que l'une produisait toujours des lésions intestinales et l'autre toujours des lésions hépatiques.

Si maintenant on considère dans leur ensemble les expériences d'immunité croisée portant sur les coccidies de divers mammifères, on peut, suivant les résultats, les grouper de la façon suivante :

\section{$1^{\circ}$ Expériences ayant abouti à des infections}

\begin{tabular}{|c|c|c|c|}
\hline Atteuns & $\begin{array}{l}\text { ESPÈCE COCGIDIENAE } \\
\text { EXPÉnIMENTÉE }\end{array}$ & $\begin{array}{c}\text { Hòte } \\
\text { NATUREL }\end{array}$ & $\begin{array}{c}\text { EsPĖCE } \\
\text { EXX'́RIMENTÉE }\end{array}$ \\
\hline $\begin{array}{l}\text { Fantham, } 1917 \ldots \ldots . . \\
\text { Rudowsky, } 1921 \ldots \ldots \\
\text { Nöller, Schürjohann } \\
\text { et Vorbrodt, } 1922 . . \\
\text { Van Nederveen, } 1923 . \\
\text { Andrews, } 1927 \ldots \ldots \ldots \\
\text { - } \\
\text { - }\end{array}$ & $\begin{array}{l}\text { Isospora belli. } \\
\text { ? "Eimeria sliedai ». } \\
\text { Eimeria faurei. } \\
\\
\text { Eimeria arloingi. } \\
\text { ? Eimeria stiedai. } \\
\text { Isospora felis. } \\
\text { Isospora rivolla. } \\
\text { Isospora felis. } \\
\text { Isospora rivolla. }\end{array}$ & $\begin{array}{l}\text { Homme. } \\
\text { Rat. } \\
\text { Mouton. } \\
\text { Chèvre. } \\
\text { Lapin. } \\
\text { Chien. } \\
\text { Chat. }\end{array}$ & $\begin{array}{l}\text { Chat. } \\
\text { Lapin. } \\
\text { Chèvre. } \\
\text { Mouton. } \\
\text { Bœuf. } \\
\text { Chat. } \\
\text { Chien. }\end{array}$ \\
\hline
\end{tabular}




\section{$2^{\circ}$ Expériences n'ayant pas abouti à une infection}

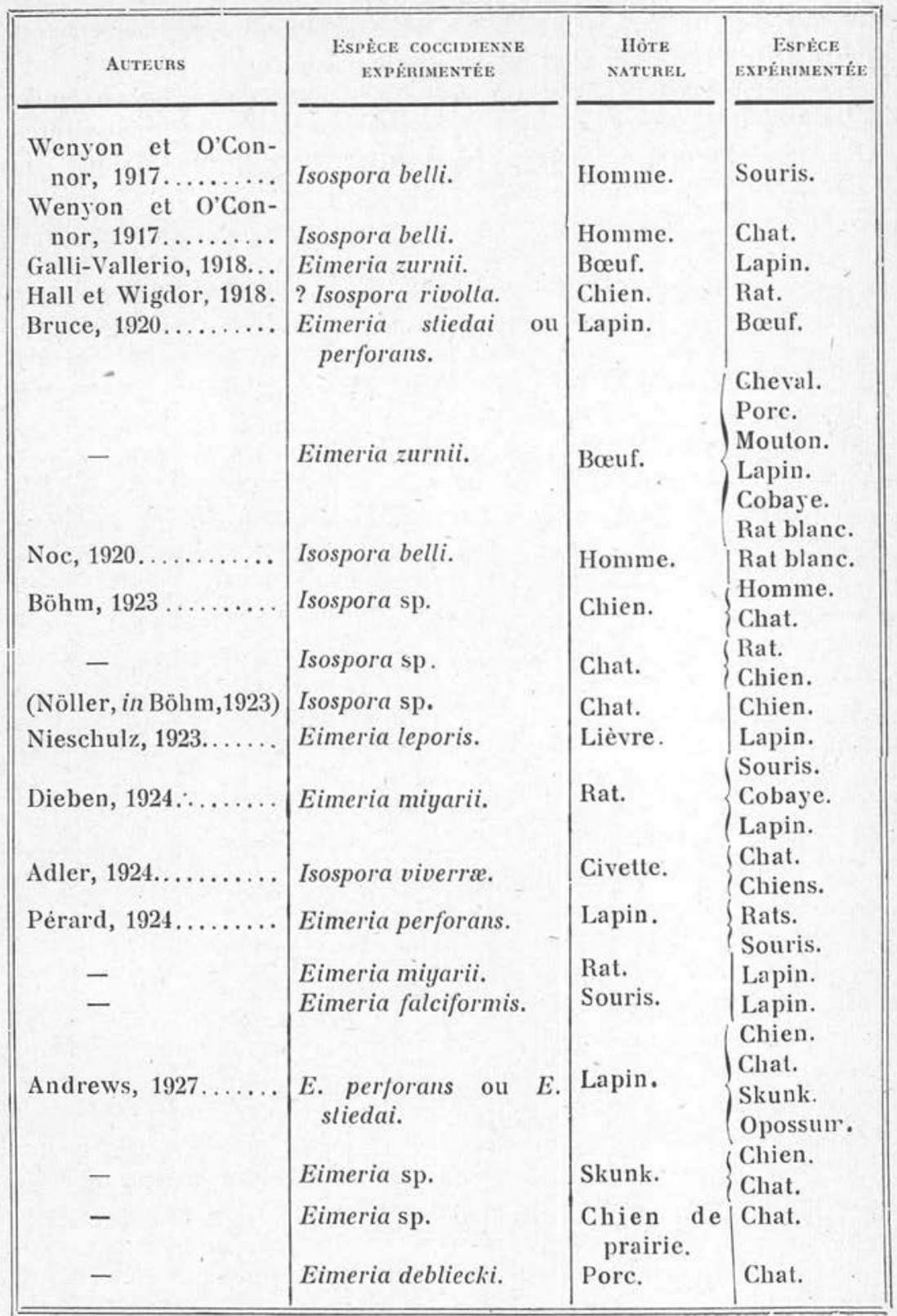


Un simple coup d'œil jeté sur les deux tableaux montre que ce sont les expériences non suivies de succès qui sont, et de beaucoup, les plus nombreuses. Mais il convient, en outre, de voir de près dans quelles conditions ont été faites les expériences signalées dans le premier tableau, afin d'en juger la valeur.

Les expériences de Fantham, qui aurait infecté des chats avec des coccidies humaines, ont été faites sans que soit écartée une infection préalable des animaux expérimentés. Dobell, puis Wenyon, en ont déjà fait la critique et il est permis de conclure avec Andrews qu'elles n'ont pas de portée. On ne saurait done les retenir.

Les expériences de Rudowsky sont également sujettes à de nombreuses critiques. Cet auteur aurait isolé chez le rat des oocystes qu'il rapportait, d'après le seul examen, à l'espèce Eimeria stiedai, parasite du lapin ; c'est là une base tout à fait insuffisante et par la suite d'autres travaux, en particulier ceux de Dieben et de Pérard, ont mieux fait connaître la coccidie du rat. Rudowsky prétendait en outre avoir avec des coccidies du rat obtenu l'infection d'un lapin ; mais son expérience a été par trop écourtée et rien ne prouve que là aussi il ne s'agissait pas d'une infection spontanée du lapin. On peut donc avec Pérard admettre qu'il n'y a pas lieu de retenir les expériences de Rudowsky.

Van Nederveen avec des oocystes des coccidies du lapin aurait infecté de jeunes veaux. Ses expériences sont loin d'entraîner la conviction et il est vraisemblable qu'il a eu, lui aussi, affaire à une infection spontanée des animaux qu'il avait utilisés.

Restent cependant deux groupes d'expériences, celles qui portent sur les coccidies du mouton et de la chèvre et celles qui portent sur les coccidies du chien et du chat. Ces expériences faites dans de bonnes conditions sont à retenir et il est légitime de considérer leurs résultats comme acquis.

On peut done conclure que, d'une façon générale, à ces deux exceptions près les coccidies de mammifères semblent être strictement spécifiques pour leurs hôtes :

En ce qui concerne les coccidies d'oiseaux, les faits acquis sont beaucoup plus rares que pour les coccidies des mammifères. Une espèce d'Eimeria, Eimeria avium, a été décrite chez la poule et l'on admet que la même espèce se rencontre chez le canard, l'oie, le faisan, le paon, le dindon, la grouse et même d'autres oiseaux ; ̀̀ vrai dire on est loin de posséder les arguments permettant d'affirmer qu'il s'agit d'une ou de plusieurs espèces. C'est ainsi que l'on admit longtemps qu'Eimeria avium pouvait également se rencontrer chez le pigeon. Les expériences récentes de Nieschulz ont montré qu'on ne pouvait infecter des poussins avec la coccidie des 
pigeons et que la coccidie de ce dernier animal devait être considérée comme due à une espèce distincte, Eimeria pfeifferi. Ce résultat montre qu'il y aurait lieu de réviser les idées actuellement admises sur les coccidies de divers oiseaux et qu'il n'est pas impossible que parmi elles on trouve la même spécificité que l'on a constatée pour celles des mammifères.

Aussi peut-on à bon droit s'étonner des résultats qu'a récemment publiés Uhlhorn ; cet auteur aurait pu en effet infecter des poussins avec des oocystes des coccidies du lapin.

L'identité de la coccidiose du lapin et du poulet peut paraître à première vue suspecte par le fait que dans la promiscuité des basses-cours ces animaux auraient les plus grandes chances de s'infecter réciproquement et que cependant les éleveurs n'ont jamais relevé d'exemple d'une épizootie commune. En outre il peut paraitre surprenant, après ce que nous avons vu de la spécificité des coccidies, qu'une coccidie de mammifère soit capable de parasiter d'emblée un oiseau.

Aussi était-il intéressant d'examiner de près dans quelles conditions avait expérimenté cet aúteur, de répéter ses expériences et de voir si ses conclusions étaient légitimes.

\section{Expériences d'Uhlhorn et critiques qu'elles soulèvent}

Le travail d'Uhlhorn est long et assez confus ; mais il donne sur son mode d'expérimentation beaucoup de détails qui sont précieux à connaître pour pouvoir ensuite l'argumenter.

Voici, fortement résumée, la technique suivie par cet auteur :

Les oocystes des coccidies étaient recueillis dans les crottes de lapins; afin d'en obtenir un plus grand nombre, Uhlhorn utilisa divers procédés d'enrichissement, mais plus particulièrement la méthode au sel de Kofoid et Barber modifiée par Fülleborn.

Les animaux qui fournirent les oocystes étaient deux jeunes lapins, àgés de 13 semaines, provenant d'un élevage où sévissait l'épizootie et où plusieurs cas de mort avaient été constatés.

La segmentation des oocystes s'obtenait en mettant les matières en mince couche dans des boîtes de Pétri. Dans ces conditions, la sporulation était terminée à partir de 41 heures.

Les poussins destinés à l'expérience étaient au nombre de 20; ils étaient de race italienne. 
Un premier lot de 10 naquit dans une couveuse qui n'avait pas cncore été utilisée ; pendant la première journée ils furent mis dans un endroit où le sol était recouvert de terreau frais de tourbe et ils furent nourris de millet. On examina au microscope un échantillon de tourbe et un échantillon de millet sans y rencontrer d'oocyste de coccidie ; ce lot fut mis ensuite dans une caisse contenant une couche de sable stérilisé par la chaleur et un peu d'ouate stérilisée pour servir d'abri aux poussins.

Le deuxième lot de 10 également, né dans les mêmes conditions que le premier, passa la première journée après l'éclosion dans une caisse qui avait servi au séjour de poussins plus âgés.

Les lots de poussins furent ensuite divisés en groupes suivant les nécessités de l'expérience. La nourriture qui leur fut donnée se composa d'œufs cuits durs hachés fins, de millet et de petit riz; plus tard ils reçurent du gruau de sarrazin, des graines de lin et d'autres grains décortiqués; l'examen microscopique des échantillons de grains n'avaient pas montré d'oocyste de coccidie; on supprima de l'alimentation toute crudité, salade ou herbe ; cela eût été, en effet, susceptible d'entraîner une contamination.

Les expériences d'infestation des poussins furent faites en déposant à l'aide d'une seringue de Pravaz à la base de la langue de ceux-ci quelques gouttes de cultures riches en oocystes; certains poussins avalèrent en outre des larves de mouches qui s'étaient développées dans les cultures; ces tentatives d'infections furent répétées à diverses dates que donne un tableau détaillé. Trois poussins conservés comme témoins n'eurent pas de repas infectants. L'examen des déjections fut ensuite fait quotidiennement pour tous les poussins pour y rechercher l'apparition éventuelle des oocystes. Dans son tableau III, p. 158-159, l'auteur donne tous les détails de l'expérience et les résultats des examens consécutifs.

Enfin, des résultats obtenus, Uhlhorn se croit autorisé à conclure qu'il a réussi à infecter des poussins avec une coccidie de lapin.

Mais avant d'accepter cette conclusion il convient de soumettre à une critique serrée la façon d'opérer de l'auteur et les bases qu'il a choisies pour directives.

Tout d'abord il ne sait de quelle coccidie il part : dans les selles de lapin, en effet, on peut rencontrer aussi bien les oocystes d'Eimeria stiedai que ceux d'Eimeria perforans, et les infections mixtes sont pour ainsi dire la règle. Uhlhorn a cru pouvoir simplement, par la taille des oocystes, distinguer les deux espèces et il se base pour cela sur les données fournies par Reichenow ; mais nous avons vu plus haut que celles-ci n'avaient qu'une valeur fort relative et il existe pour les distinguer des moyens beaucoup plus sûrs 
qu'a fourni Pérard, auteur dont Uhlhorn semble complètement ignorer le travail cependant fort important et très antérieur au sien. Il y avait pourtant possibilité d'obtenir une souche pure d'oocystes en s'adressant à la coccidiose hépatique et l'on se demande pourquoi Uhlhorn n'y a pas songé.

Quant à sa technique de sporulation il est un détail qui doit immédiatement arrêter l'attention : les cultures n'étaient pas à l'abri de contaminations accidentelles en particulier par les mouches; or l'expérience se faisait dans un élevage de volailles, à Crollwitz, et les mouches peuvent dans ces conditions être un moyen de dissémination de la coccidie des poules. Il est vrai que l'auteur dit qu'à cet élevage de volailles on n'avait pas observé de coccidiose des poussins mais il ajoute plus loin que les animaux plus âgés n'étaient pas " entièrement libres de coccidies " (p. 126).

Bien mieux, il semble que l'auteur ait cherché à réaliser cette contamination des cultures car il dit textuellement : "Les oocystes qui s'étaient segmentés le mieux et le plus abondamment étaient ceux contenus dans des boîtes de Pétri dont le couvercle était endommagé, de sorte que les matières fécales n'étaient pas complètement à l'abri, quelques mouches y étaient entrées et avaient déposé leurs œufs " (p. 126). Les larves se développaient par la suite et l'auteur juge que leur présence avait une action bienfaisante par l'aération des cultures. Cette manière de procéder suffirait à elle seulè à infirmer les résultats que l'auteur prétend avoir obtenus, si le simple examen du tableau III où il donne tous ses résultats d'expérience ne montrait que les 3 poussins témoins qui n'avaient pas reçu de cultures ont cependant présenté des oocystes dans leurs excréments à peu près en même temps que les poussins expérimentés.

Des expériences d'Uhlhorn on ne peut donc pas tirer la conclusion qu'il en donne lui-même mais la suivante: l'infection spontanée n'a pas été écartée; il est fort vraisemblable que les oocystes observées chez les animaux expérimentés et que Uhlhorn rapporte à la coccidie du lapin doivent être rapportées à la coccidie des poules.

Ainsi l'examen critique de l'expérience d'Uhlhorn ayant montré que celle-ci était sans valeur, il était intéressant de la reprendre avec une technique plus précise. Nous allons maintenant exposer les résultats que nous avons obtenus dans cet ordre d’idées. 


\section{Reprise des expériences d'Uhlhorn}

Notre expérience fut réalisée dans les conditions suivantes :

Nous avons pu nous procurer aux Halles Centrales des foies de lapins parasités par Eimeria stiedai. Nous avons recueilli toutes les nodosités blanches desquelles nous avons extrait, par e̋crasement dans de l'eau distillée, un liquide épais constitué par des oocystes d'Eimeria stiedai et des cellules épithéliales des canaux biliaires.

Après décantation nous avons fait segmenter ces oocystes dans une solution d'acide chromique à 1 pour 1.000 .

La première culture a été effectuée le 14 mai ; la segmentation était terminée le 18 mai.

La deuxième culture a été effectuée le 19 mai ; la segmentation était terminée le 24 mai.

La troisième culture a été effectuée le 22 mai ; la segmentation était terminée le 26 mai ; une quatrième culture effectuée le 31 mai n'a pas donné de segmentation.

Nous avons choisi 24 poussins, reçus 5 heures après leur naissance d'œufs qui avaient été couvés artificiellement. Ils n'avaient encore absorbé aucun aliment. Comme nourriture nous leur don. nions une pâtée composée de farine de maïs, farine d'orge, chapelure de pain, poudre de viande, poudre d'os, coquilles d'huîtres, phosphate de chaux.

L'expérience a débuté le 25 mai, jour de la naissance des poussins. Ceux-ci ont été répartis en deux groupes de douze isolés dans deux petits parcs séparés l'un de l'autre et entourés d'un grillage. Chacun d'eux contenait, outre les appareils nécessaires à la nourriture, une éleveuse, chauffant à $23^{\circ}$ environ. Nous n'avons expérimenté que sur un groupe de 12 poussins (groupe A) conservant l'autre (groupe B) comme témoin.

Le 25 mai, vers 4 heures, nous avons fait ingérer aux poussins du groupe A les premiers aliments auxquels nous avons mélangé la culture du 14 mai. Le lendemain matin, à l'examen des selles frâ̂ches, on trouvait pour le groupe A quelques oocystes intacts, les déjections du groupe $B$ ne présentant rien. Le 26, au soir, nouvelle ingestion des aliments mélangés à la culture du 19 mai. Le 27, même présence d'oocystes non altérés dans les selles fraîches du groupe A ; rien pour le groupe B. Le 28, pas d'oocystes à l'examen des déjections. Le 29, nouvelle ingestion d'oocystes segmentés de la culture du 22 mai, cette fois l'ingestion est faite directement à la pipette, après avoir bien lavé les oocystes segmentés pour les 
débarrasser de l'acide chromique. Chaque poussin du groupe A absorbe environ 15 à 20 gouttes d'une culture contenant 8 à 10 oocystes par champ (objectif 4 ).

Le lendemain 30 mai, on constate, comme pour les deux premières ingestions, la présence d'oocystes non altérés. La moitié de la même culture avait été conservée pour la faire ingérer par la suite à un lapin témoin.

Du 30 mai au 14 juin les poussins n'ont pas absorbé de nouveaux repas infectieux. Pendant ces quinze jours ils n'ont présenté aucune coccidie dans leurs déjections ; rien ne fut modifié dans leur état, nous n'avons constaté aucun des symptômes décrits par Railliet et Lucet dans la coccidiose du poulet: ni tristesse, ni diarrhée, ni constipation. Les plumes restent aussi brillantes et croissent de même chez l'élevage témoin et l'élevage en expérience. L'activité des uns et des autres est identique; aucun cas de mort ne s'est produit dans les deux groupes.

Le 7 juin, nous avons commencé une nouvelle culture d'oocystes de Eimeria stiedai pris comme antérieurement dans des foies de lapins bien parasités.

Le 12 juin, les oocystes sont segmentés, nous attendons, comme pour les cultures précédentes, un jour ou deux de plus afin que la segmentation soit bien complète. Le 14 juin, nous faisons ingérer cette nouvelle culture au groupe A, à l'aide d'une pipette ; nous donnons comme précédemment 15 à 20 gouttes par poussin d'une dilution contenant environ 12 à 14 oocystes par champ (objectif 4 ). Le 15 juin, présence d'oocystes intacts dans les selles des poussins du groupe A. Les 16,17 et 18 juin, rien dans les deux élevages. Les poussins conservent leur mème activité, cependant l'un d'eux meurt dans le groupe B, c'est-à-dire dans l'élevage témoin. Le 19, aucun oocyste n'est trouvé dans les déjections du groupe A.

Le 22, rien n'est changé dans les deux groupes de poussins.

Afin de contrôler ces premières expériences, nous avons voulu, comme nous le disons un peu plus haut, infecter un lapin indemne de toute coccidiose. A cet effet, nous avons pu nourrir au biberon deux jeunes lapins de 15 jours non encore sevrés (nous conseillons pour que ces lapins nourris au biberon puissent vivre que le lait qu'on leur donne provienne toujours de la même vache). Peu après trois semaines, ces lapins mis séparément dans deux cages grillagées et flambées préalablement pour les stériliser, ont été nourris avec des légumes préalablement lavés puis essorés. Malheureusement malgré toutes les précautions il ne nous a pas été possible d'empêcher une infection spontanée et à la fin du premier mois, 
leurs crottes contenaient des oocystes de coccidies mesurant de 26 à $38 \mu$.

Néanmoins, il était intéressant de tenter de provoquer chez l'un d'eux une infection suraiguë. C'est pourquoi, le 10 juin, nous faisions ingérer par l'un de nos lapins la culture réservée à cet effet. Le 8 juillet, le lapin meurt. A l'autopsie, nous trouvons un gros ventre, un foie augmenté de volume et bourré de nodosités blanchâtres caractéristiques; le cholédoque et les autres canaux sont hypertrophiés ; la vésicule biliaire mesure $4 \mathrm{~cm}$., 5 de longueur, elle est bourrée d'oocystes d'Eimeria stiedai. Nous sacrifions le même jour le lapin témoin et nous trouvons comparativement les chiffres suivants :

Lapin témoin

Poids total.

Poids du foie............
$1.665 \mathrm{gr}$.

$83 \mathrm{gr}$.
Lapin infecté

Poids total........... $1.400 \mathrm{gr}$. $185 \mathrm{gr}$.

Il nous est donc facile de constater une infection suraiguë chez notre lapin infecté. L'aügmentation considérable du poids du foie, la diminution du poids total de ce lapin cachectisé, la présence d'un nombre considérable d'oocystes dans une vésicule biliaire hypertrophiée et dans toutes les nodosités hépatiques sont la signature d'une infection suraiguë provoquée par l'ingestion de nos oocystes segmentés. Nous constatons enfin que c'est bien vers le foie que s'est portée l'infection intense révélée par l'autopsie. Eimeria stiedai est donc spécifique pour le foie du lapin.

En résumé, nous avons done fait ingérer à douze poussin 3 5 cultures bien segmentées d'oocystes d'Eimeria stiedai. Le temps consacré à l'expérience a été de 32 jours. Si nous avions dû avoir une infection, elle se serait produite beaucoup plus rapidement. De plus, étant donnée la grande facilité avec laquelle le poussin peut s'infecter quand il s'agit d'Eimeria avium, et l'extrême gravité de la coccidiose qu'il présente, au moins un cas de mort se fût produit dans le groupe expérimenté. Or, le 8 juillet, nous ne constatons aucune infection à coccidies chez nos poulets.

\section{RÉSUMÉ}

L'ingestion expérimentale par des poussins âgés de 1 à 32 jours (c'est-à-dire éminemment réceptifs à une infection coccidienne) de très nombreux oocystes entièrement segmentés d'Eimeria stieciai 
n'a déterminé aucune infection. Il y a seulement élimination au cours de la première journée de kystes non altérés.

On ne peut donc accepter les résultats de Uhlhorn qui aurait réalisé facilement cette infection. L'examen détaillé des condilions dans lesquelles cet auteur a opéré montre en effet qu'il était loin d'avoir éliminé toute cause de contamination accidentelle.

Rien ne permet donc à l'heure actuelle de conclure à l'identité de la coccidie hépatique du lapin et de la coccidie intestinale du poulet.

\section{Bibliographie}

Andrews (J.-M.). - Host-Parasite Specificity in the Coccidia of Mammals. Journal of Parasilology, XIII, 1927, p. 183.

Basset (J.). - La coccidiose intestiuale, maladie des jeunes animaux. Recueil de Médecine vétérinaire, LXXXVI, 1909, p. 463.

Bӧнм (L.-K.). - Morphologische und experimentelle Bciträge zur Kenntniss der Hunde und Katzenkokzidiose. Wiener tierärlzliche Wochenschrift, X, 1923, p. 140.

Bruce (E.-A.). - Bovine Coccidiosis in British Columbia. Journ. Amer. Vet. Med. Assoc., LVIII, 1920, p. 633.

Brumpt (E.). - Précis de Parasitologie, 4e édition. Paris, Masson, 1927.

Dieben (C.-P.-A. - Over de Morphologie en Biologie van het Rattencoccidium Eimeria nieschulzi $n$. sp. en zijne Verspreiding in Nederland. Thèse Doctorat vétérinaire, Utrecht, 1924.

Dobert (C.). - A revision of the Coccidia Parasitic in Man. Parasilology, XI, 1919, p. 147.

Fantham (H.-B.). - Protozoa in The Animals Parasiles of Man par Fantham, Stephens et Theobald. Londres, 1916.

Galdi-Valerio (C.). - Notes de parasitologie et de technique parasitologique. Schweiz. Arch. für Tierheilk., 1919, p. 289.

Hall (M.-C.) et Wigdor (M.). - Canine Coccidiosis with a Note Regarding Other Protozoa Parasites from Dog. Journ. Amer. Vel. Med. Assoc., LIII, 19:8, p. 64.

Neumann (L.-G.). - Parasiles et maladies parasitaires des oiseanx domestiques. Paris, Asselin et Houzeau, 1909, p. 109.

Noc. - Nouveau cas de coccidiose intestinale humaine à Isospora. Bull. Soc. de Path. exot., XIII, 1920, p. 785.

Nöller, Schurjohann et Vorbrodt. - Zur Kenntniss der Ziegen-und Schafkokzidiose. Berliner tierärtztl. Woch., XXXVIII, 1922, p. 193.

PÉrand (C.). - Recherches sur les coccidies et les coccidioses du lapin. C. R. Ac. des Sc., CLXXVIII, 1924, p. 2131.

- Recherches sur les coccidies et les coccidioses du lapin. Annales de rInstitut Pasteur, XXXVIII, 1924, p. 953 et Thèse Doct. vétérinaire Paris, 1925. Paris, Marétheux, 72 p.

1. Recherches sur les coccidies et les coccidioses du lapin. II. Contribution à l'étude de la biologie des oocystes de coccidies. Anna'es de l'Institut Pasteur, XXXIX, 1925, p. 505. 
PÉraRd (C.). - Recherches sur les coccidies et les coccidioses du lapin. III. Etude de la multiplication endogène. Annales de l'Institut Pasteur, XXXIX, 1925, p. 952 .

Railliet (A.) et Lucet (A.). - Développement expérimental des coccidies de l'épithélium intestinal des lapins et de la poule. C. R. Soc. de Biologie, XLIII, 1891, p. 820.

- Note sur quelques espèces de coccidies encore peu étudiées. Bull. Soc. Zool. de France, 1891, p. 246.

Reichenow (E.). - Die Coccidien. Handbuch der pathogenen Prolozoen, $8 \circ$ Lief., Leipzig, Ambrosius Barth, 1921, p. 1253.

Rudov́sky (F.). - Die Kokzidiose der Wanderratte (Mus decumanus Pall.) und ilıre Beziehung zur Kaninchenkokzidiose. Centralblatt f, Bakt., I, Orig., LXXXVII, 1921, p. 427.

Uhцноп (Е.). - Uebertragungsversuche von Kaninchencoccidien auf Hühnerkücken. Archiv für Prolislenkunde, LV, 1926, p. 101.

Van Nederveen (H.-J.). - Infectieproef van het Rund met Konijnencoccidien. Tijdschr. van Vergelijkende Geneesk. enz., VIII, 1923.

Wenyon (C.-M.). - Coccidiosis of Cats and Dogs and the Status of Isospora in Man. Ann. Trop. Med. and Par., XVII, 1923, p. 231.

- Protozoology ; a Manual. Lon'dres, Baillière, Tindall et Cox, 1926, II, p. 830 et sq.

Laboraloire de parasilologie de la Faculté de médecine de Paris 